\title{
Solar impulsive EUV and UV brightenings in flare footpoints and their connection with $\mathrm{X}$-ray emission
}

\author{
T. Mrozek ${ }^{1}$, M. Tomczak ${ }^{1}$, and S. Gburek ${ }^{2}$ \\ 1 Astronomical Institute, University of Wrocław, ul. Kopernika 11, 51-622 Wrocław, Poland \\ e-mail: mrozek@astro.uni.wroc.pl \\ 2 Space Research Centre, Polish Academy od Sciences, Solar Physics Division, ul. Kopernika 11, 51-622 Wrocław, Poland
}

Received 16 April 2007 / Accepted 14 June 2007

\section{ABSTRACT}

\begin{abstract}
Context. The thick-target model of the impulsive phase of solar flares describes the propagation of non-thermal electron beams into the chromosphere. The stopping depth of the electron is related to its energy. Thus, the thermal reaction at different levels should be connected to the parameters of the electron spectrum.

Aims. We investigate the impulsive thermal reaction produced by non-thermal electron beams at different heights of the solar atmosphere observed in solar flare footpoints.

Methods. We used methods developed for the investigation of the impulsive SXR brightenings. The EUV and UV images obtained by TRACE in the $171 \AA$ and $1600 \AA$ filter were used as the basic data set for this analysis. For the first time, EUV images have been desaturated using diffraction pattern properties.

Results. We find that the thermal impulsive reaction observed in solar flare footpoints is different for UV and EUV brightenings. Results are compared to those obtained for the impulsive SXR brightenings. We find that observed dissimilarities are connected to the properties of the non-thermal electron spectrum and to the depth of their penetration into the chromosphere. Moreover, we show that the lower levels of the chromosphere are excited by more energetic electrons.
\end{abstract}

Key words. Sun: flares - Sun: UV radiation - Sun: X-rays - gamma rays - Sun: chromosphere

\section{Introduction}

The thick-target model (Brown 1971) gives a satisfactory explanation of processes observed in flare footpoints during the impulsive phase. It is assumed there that electrons are accelerated at the top of the flaring loop and after escape from the acceleration region they propagate along field lines. A part of them moves downward to the chromosphere where they deposit their energy due to Coulomb collisions. During this process we observe hard $\mathrm{X}$-ray (HXR) brehmsstrahlung and the thermal reaction of the plasma in UV, EUV, soft X-ray (SXR), and even white light.

According to this model we expect a relation between the kinetic energy of electron and the column depth. This relation was observed directly by measuring the height of HXR footpoint sources in several energy ranges (Matsushita et al. 1992; Aschwanden et al. 2002; Mrozek 2006). Moreover, the effect of the energy-height relation in the thermal reaction of the solar atmosphere should be observed. Roughly speaking, we expect that electrons propagating through the solar atmosphere excite successive levels emit SXR, EUV, UV, and white-light.

The impulsive SXR brightenings in solar flare footpoints have been discovered by Yohkoh (Hudson et al. 1994) and their close relationship with HXRs investigated (Tomczak 1999; Mrozek \& Tomczak 2004, hereafter Paper I). It was shown in Paper I that close temporal and spatial relationships between HXR emission and SXR brightenings suggest a common physical cause - the non-thermal electron beam. Moreover, the impulsive response in flare footpoints observed in the SXR and HXR images were quantitatively compared. The authors observed the relation between the relative productivity of SXRs with regard to
HXRs and the power-law index of the photon flux. This relation is a function of the energy of HXR photons. They found that the impulsive SXR brightenings are caused by relatively lowenergy electrons. Electrons with higher energies should reach lower, denser parts of the solar atmosphere (Farnik et al. 1997) and produce the impulsive reaction seen in EUV and UV radiation.

Early analysis of UV and EUV radiation from the Sun was based on sudden ionospheric disturbances (SID) detected in the Earth's atmosphere (Donnelly 1970). The direct comparisons (Emslie et al. 1978; Kane et al. 1979) showed that pulses observed in UV and EUV are delayed relative to HXR pulses less than 5 s. Observations obtained with the Solar Maximum Mission, SMM showed that this relationship can be even closerpulses in both kinds of radiation were observed on $1 \mathrm{~s}$ time scales (Cheng et al. 1988). Such a short delay suggests that EUV and UV radiation is produced directly by non-thermal electrons.

The investigations described above were made without spatial resolution. In 1998 the Transition Region and Coronal Explorer (Handy et al. 1999) TRACE, was launched and gave a possibility for analysing EUV and UV images with high spatial and temporal resolution. Fletcher \& Hudson (2001) show that HXR emission sources and EUV (195 A) ribbons are observed in the same locations with spatial accuracy equal to about $3^{\prime \prime}$. Moreover, they show that HXR lightcurve has a similar shape to the EUV lightcurve obtained for footpoints. The authors suggest that such a relation is strong support for the scenario in which EUV emission is produced mainly by a non-thermal electron beam. Their analysis of EUV and HXR signals was based on the qualitative comparison between lightcurves. There was no 
Table 1. List of investigated flares.

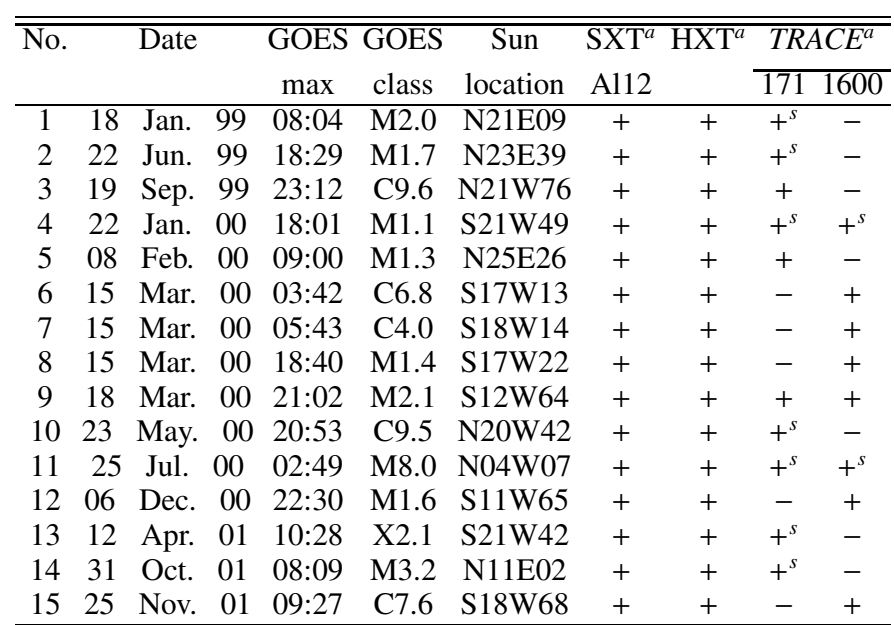

${ }^{s}$ Saturated sources. ${ }^{a}$ Plus means that the event was observed with: SXT/Al12, HXT, TRACE $171 \AA$, and TRACE $1600 \AA$ A, respectively.

quantitative analysis of fluxes because of the saturation of EUV sources.

Warren \& Warshall (2001) have analysed several flares observed by TRACE in $1600 \AA$ filter. Lightcurves of individual footpoints visible in the UV were compared to HXR lightcurves obtained by the Yohkoh Hard X-Ray Telescope, HXT and the Burst and Transient Source Experiment (BATSE) installed onboard the Compton Gamma-Ray Observatory. They show that there are two kinds of UV footpoint sources. The first group shows UV brightenings before the HXR onset, the second is spatially correlated with HXR sources, and UV brightenings are observed simultaneously with HXR bursts.

Recently, Alexander \& Coyner (2006) have analysed the 2002 July 16 flare observed simultaneously by TRACE and RHESSI. The authors analysed six UV kernels observed during the impulsive phase of the flare. The UV lightcurves were strongly correlated with HXR time profile, which suggests common physical cause for both types of emission. The HXR source $(25-100 \mathrm{keV})$ was observed only in the southern footpoint, while the northern one showed no significant HXR emission. In the lower energy range (6-25 keV), a clear loop was observed what suggests that there was a magnetic connection between the southern and northern footpoints, even if there is no noticeable HXR emission in the northern footpoint.

In this paper we present an analysis of several flares observed in EUV and UV ranges. For the analysis of the impulsive UV and EUV brightenings, we used the method developed for investigating the impulsive SXR brightenings (Paper I). The qualitative analysis is based on the investigation of individual bursts of EUV, UV, and HXR emissions. For the first time, quantitative analysis of spatially resolved EUV, UV, and HXR emissions was made.

Using different instruments observing the Sun, we were able to make an investigation of some properties of solar footpoints at several levels of the atmosphere. In Paper I we investigated impulsive SXR brightenings that occurred in the lower corona. In this paper we use TRACE $171 \AA$ and $1600 \AA$ filters to observe radiation from the flare transition region and lower chromosphere, respectively. Altogether, this set of data gave us an opportunity to investigate the thermal reaction on non-thermal electron beams in solar flare footpoints at three different levels of the atmosphere.

The paper is organised in the following way. Section 2 contains a short description of TRACE and the filters used. Moreover, it contains description of the method used for the desaturation of the signal detected with the $171 \AA$ filter. The analysis techniques are presented in Sect. 3. Section 4 contains description and discussion of the obtained results. The conclusions and summary are given in Sect. 5 .

\section{Observations and data reduction}

For the analysis of UV and EUV impulsive brightenings, we used data obtained with the TRACE telescope (Handy et al. 1999). To investigate the response in EUV and UV ranges we chose images taken with the $171 \AA$ and with the $1600 \AA$ filter, respectively. We do not analyse data obtained with the $195 \AA$ and $284 \AA$ filters. The main reason is the small number of images obtained with the use of these filters.

The $30 \mathrm{~cm}$ Cassegrain telescope installed onboard TRACE gives the $1^{\prime \prime}$ resolution $\left(0.5^{\prime \prime}\right.$ pixel $)$ with the $8.5^{\prime} \times 8.5^{\prime}$ field of view. Wavelength selection is managed using the system of filter wheels that makes it possible to observe the Sun in different bands from white light to EUV. Images are taken with the CCD coated with the lumogen thereby increasing the efficiency of the detector for the UV and the EUV radiation (Deeg \& Ninkov 1995; Kristianpoller \& Dutton 1964).

The hard X-ray lightcurves and images were obtained using the Hard X-ray Telescope, HXT (Kosugi et al. 1991) onboard the Yohkoh satellite (Ogawara et al. 1991). The HXT was a Fourier synthesis imager that observed the whole Sun in four energy bands: 14-23 keV (L channel), 23-33 keV (M1), 33$53 \mathrm{keV}(\mathrm{M} 2)$, and 53-93 keV (H). The temporal resolution for lightcurves is $0.5 \mathrm{~s}$ in the flare mode. Image formation time scales depend on the count statistics. The spatial resolution of the HXT images was estimated to be about $5^{\prime \prime}$.

For selection of events we used the catalogue by N. Nitta ${ }^{1}$. In a preliminary selection we looked for time intervals covering impulsive phases of flares, for which all instruments provided data, and we found 56 events. However, for the majority of them TRACE did not observe the flaring region, reducing the number of analysed flares to 15. All of them are shown in Table 1, which shows that there are only three events observed with all instruments/filters simultaneously. We have included the SXT/A112 observations, to make certain that the impulsive SXR brightenings for analysed events are similar to the ones described in Paper I.

Almost all images of the impulsive phase observed by TRACE were saturated due to the 12-bit saturation level of the Analog to Digital Converter (ADC), which is related to $20 \%$ capacity of the CCD pixel (full well). For this reason we were able to investigate the TRACE $1600 \AA$ images obtained for only weak flares. For the TRACE $171 \AA$ images we recovered a saturated signal using the method based on the diffraction pattern caused by the mesh supporting the EUV filters. As there is no mesh supporting the TRACE $1600 \AA$ filter, we did not observe diffraction sources, which would enable us to recover saturated signal.

Lin et al. (2001) have made a detailed analysis of the TRACE diffraction pattern for $171 \AA$ filter and compared results with observations. They calculated how the signal in the diffraction

\footnotetext{
1 http://www.lmsal.com/nitta/sxt_trace_flares/ list.html
} 
Table 2. Diffraction pattern properties for the zeroth, the first and the second diffraction orders. ${ }^{a}$

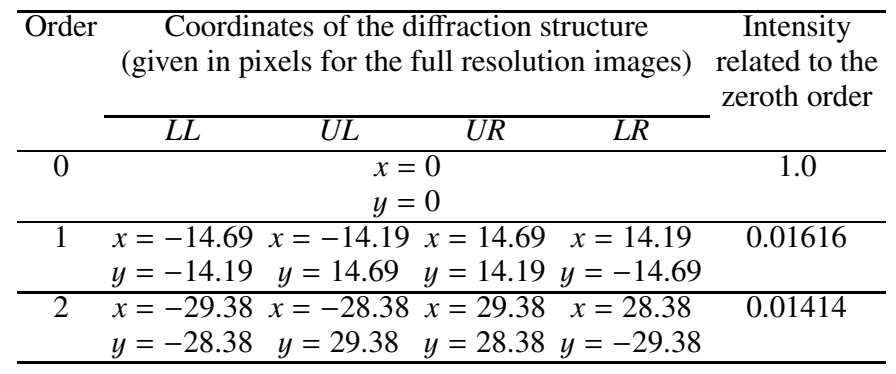

${ }^{a}$ Lin et al. (2001), Gburek et al. (2006).

structure of a given order is scaled relative to the signal located at the zeroth-order position. This scaling law can be used for recovering the true signal in saturated pixels taken for analysis.

The observed diffraction pattern is built up from diffraction sources located in the arms of the diffraction cross, which we identify with the following code: LL-Lower Left, LR-Lower Right, UL-Upper Left, UR-Upper Right. By adding numbers related to the diffraction order, we are able to identify a particular diffraction source; e.g., UL2 means the diffraction source of the second order located in the upper left arm of the diffraction cross. The parameters of the diffraction pattern used in our analysis are presented in Table 2.

Gburek et al. (2006) analysed the geometry of the TRACE diffraction pattern. In particular, they showed that structures seen in low diffraction orders are almost exact copies of the zerothorder structure scaled by a multiplicative constant and shifted by an appropriate translation vector. Hence, even if the signal in the zeroth order is saturated, it can be recovered from the signal recorded in low diffraction orders.

In this paper we have applied these results to recover the signal in saturated pixels. First, we determined the shape of the emission source using the best-resolved, background-subtracted diffraction structure seen in the first or the second order of diffraction. Next, we shifted this shape to the zeroth order to obtain the borders of the saturated source. The signal of each saturated pixel within the borders was then recovered using the scaling factors in Table 2, which depend on the diffraction order. An estimate was determined for each of the diffraction sources seen in the first and second orders. This way we obtained eight estimations of the value in the zeroth order. Diffraction sources can overlap other structures seen in the image, which overestimates the real value in the zeroth order pixel. For this reason we calculated the mean value only of the values for which the standard deviation was less than $3 \sigma$. We consider this mean value as the final estimation of the signal in the zeroth order.

An example of the signal distribution in saturated sources before and after the signal recovery is presented in Fig. 1. Lower panels present the same image as a surface plot. It shows that, after desaturation, the signal in the zeroth order can even be ten times higher.

\section{Analysis}

Investigation of the impulsive UV and EUV brightenings is based on the method developed in Paper I with one exception. For impulsive SXR brightenings we prepared a numerical routine in Paper I to extract pixels showing impulsive changes of the intensity by analysing their light curves. That routine cannot be applied to TRACE observations due to a great number of emitting structures that, although they are not footpoints, can change their brightness rapidly. Examples of such features are the diffraction sources seen in Fig. 1 showing impulsive brightenings, although they are not solar structures.

Hence, for each flare in Table 1, we defined bright kernels of UV or EUV emission using the isophote of the 10\%-30\% of the brightest pixel. Usually, in TRACE images, taken during the impulsive phase, the footpoints are the brightest structures, and they contain $70 \%-90 \%$ of the signal.

To obtain the observational characteristics of UV or EUV impulsive brightenings, we considered each kernel seen in TRACE images taken during the impulsive phase. The light curves of each kernel were compared to the HXT light curves. We defined several observational characteristics of impulsive UV and EUV brightenings as follows:

1. Full width at half maximum (FWHM) duration time, $\Delta t-$ the time interval during which the UV or EUV signal was above half of the maximum value,

2. delay, $t-t_{\mathrm{HXR}}-$ the time difference between the maxima of the UV or EUV light curve and the HXR light curve,

3. $\Delta t / \Delta t_{\mathrm{HXR}}$ is the relative duration of the UV or EUV $F W H M$ duration with respect to the HXR one, which can be interpreted as the relative duration of the response to the nonthermal electrons in both domains.

This part of analysis was done with total HXR lightcurves. We tried to obtain HXR lightcurves for each footpoint separately; however, the temporal resolution of such lightcurves was not satisfying due to photon statistics. For the strongest events we were able to achieve resolution of the order of $10 \mathrm{~s}$. Since the temporal resolution of TRACE images was not high either, we decide to use total HXR lightcurves for minimizing errors. Only for one flare were we able to reconstruct images with $2 \mathrm{~s}$ temporal resolution. This event (No. 9, Table 1) will be discussed further.

For the quantitative analysis we selected UV/EUV emission kernels that were spatially correlated with HXR sources observed simultaneously by the HXT. Typically, we observed several small kernels of UV or EUV emission that were correlated with one larger HXR source. It is caused by much higher angular resolution of HXT (5") than the TRACE image resolution ( $\left.1^{\prime \prime}\right)$. Recent observations using RHESSI shows that HXR sources are more complex in structure if observed with better spatial resolution. Liu (RHESSI Science Nuggets ${ }^{2}, 2006$ ) described observation of HXR ribbons revealing several sources well-connected to ribbons observed by TRACE $1600 \AA$.

For the flux comparison, we therefore considered all UV or EUV kernels observed close to the particular HXR source. For these UV or EUV kernels, we observed impulsive brightenings within the period of the main HXR burst or several seconds later. We summed up signals from these maxima and treated them as UV or EUV responses. The borders of the HXR source were defined with the use of images reconstructed for all available HXT channels and for the time interval covering the strongest HXR burst. Images were reconstructed with the use of the MEM-Sato method (Sato et al. 1999). Moreover, we calculated the UV or EUV and HXR responses of complete events. By response of a complete event, we mean the background-subtracted signal obtained due to integrating entire UV or EUV images for a particular time interval. Similarly by a response of HXR complete

\footnotetext{
2 http://sprg.ssl.berkeley.edu/ tohban/nuggets/ ?page=article\&article_id=40
} 

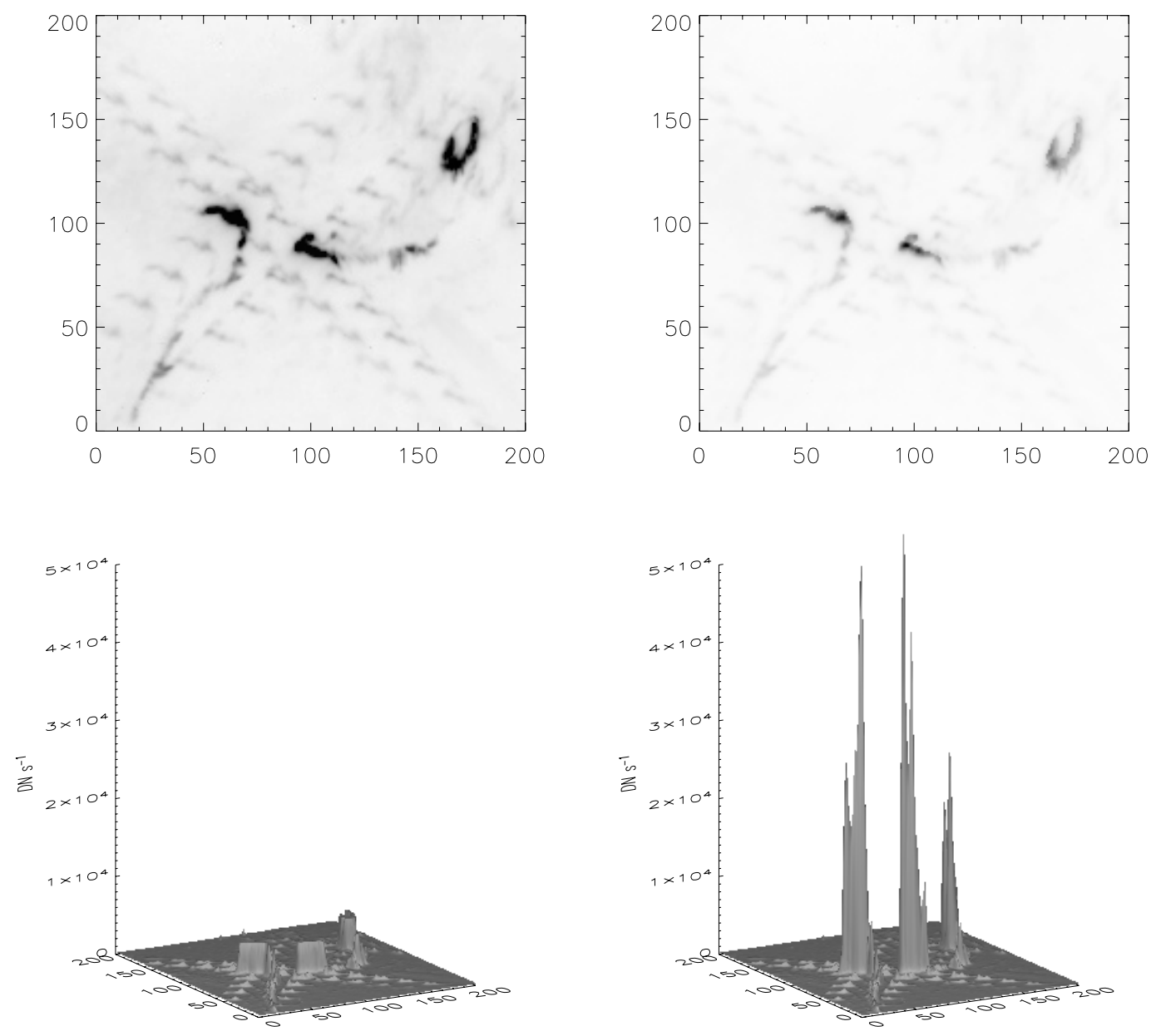

Fig. 1. An example of the desaturation method for the TRACE $171 \AA$ image. The left panel presents the original signal distribution. In the right panel, the signal has been recovered using the diffraction pattern properties. Lower panels present the surface representations of images above.

event, we mean total signal obtained in a particular channel and time interval. The complete event fluxes were used to control whether there is any systematical difference occurring between the results obtained for spatially resolved sources and complete events.

Usually, a pointing of TRACE images should be corrected before comparing them with HXT images. We used two methods based on the cross-correlation between TRACE $171 \AA$ images and Extreme ultraviolet Imaging Telescope (EIT) images taken in the same energy band ${ }^{3}$ or between white-light TRACE and Michelson Doppler Imager (MDI) continuum images ${ }^{4}$. These methods give an accuracy of the image coalignment of the order of $5^{\prime \prime}$, which is good enough for determining which UV/EUV source is related to the particular HXR footpoint. Examples of the relative position of UV or EUV kernels and HXR emission sources are presented in Figs. 2 and 3.

\section{Results}

In summary, for the 15 flares listed in Table 1, we found 26 impulsive EUV brightenings and 42 impulsive UV brightenings. Examples of light curves for the impulsive brightenings

\footnotetext{
${ }^{3}$ http://hesperia.gsfc.nasa.gov/ ptg/trace-align/

${ }^{4}$ http://www. cora.nwra.com/ ${ }^{\text {metcalf/TRACE/pointing.html }}$
}

observed in the TRACE $171 \AA$ and TRACE $1600 \AA$ are presented in Figs. 4 and 5, respectively.

\subsection{TRACE $171 \AA$ : observational characteristics of impulsive EUV brightenings}

Usually, TRACE images for different flares have different temporal resolutions depending on the observational sequence. From observations with the $171 \AA$ filter, we chose the ones obtained with temporal resolution better than $20 \mathrm{~s}$. This upper limit is relatively high, but models of EUV emission produced during non-thermal electron beams show that EUV brightening typically lasts even several dozen seconds (Somov et al. 1981). Observational characteristics for 26 impulsive events were collected and presented in Fig. 6 in the form of normalized histograms.

The left panel in Fig. 6 presents $F W H M$ duration times, $\Delta t_{\text {EUV }}$, of the impulsive EUV brightenings for spatially resolved footpoints seen in the TRACE $171 \AA$ images. About $70 \%$ of the values are in the 20-80 s interval and are very similar to the duration times for the impulsive SXR brightenings presented in Paper I. The result is rather surprising since the radiative losses of the $1 \mathrm{MK}$ plasma are more than 10 times larger than for 10 MK plasma. Hence, we expected a shorter duration for the 


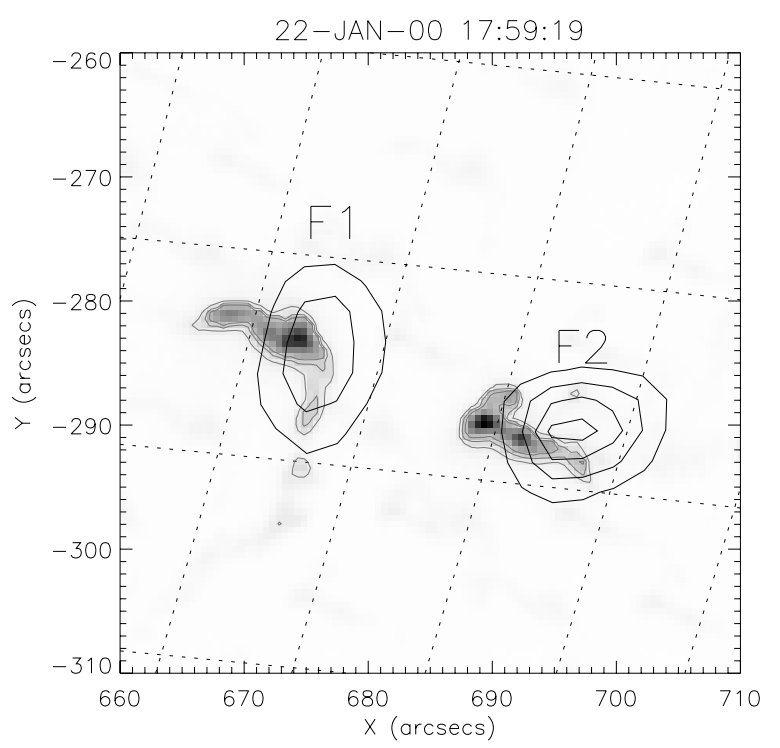

Fig. 2. The image of the 22 Jan. 00 flare obtained at the maximum of its HXR light curve. The gray scale represents the TRACE $171 \AA$ emission distribution. Black contours - HXR emission at 30, 50, 70, and $90 \%$ of the maximum value. Gray contours - EUV emission at 10 and $30 \%$ of the maximum value.

impulsive EUV brightenings. On the other hand, long-lasting EUV brightenings can be real due to the following reasons.

Numerical models of footpoints heated by non-thermal electron beams (Nagai \& Emslie 1984; Fisher et al. 1985a-c) show that the layer of temperature of $10^{6} \mathrm{~K}$ is the most suddenly evolving part of the solar atmosphere during the impulsive phase of the solar flare. It is heated both by the non-thermal electron beam and the conductive flux coming from neighbouring hot plasma (about $10^{7} \mathrm{~K}$ ) present in the footpoint just above the layer emitting in the EUV range. Such conductive flux is always present in the theoretical models and should not be mixed up with the conductive flux coming from the top of the flaring loop. We roughly expect that this conductive front should be present as long as the impulsive SXR brightening is observed. In numerical models, it propagates no more than $1000 \mathrm{~km}$ downward, so we do not observe EUV footpoints changing their vertical position since it happens at a distance comparable to the spatial resolution of the TRACE telescope.

Another important explanation of the long duration of EUV brightenings is the response function of the $171 \AA$ filter. It has recently been shown that the high-temperature plasma (about $10^{7} \mathrm{~K}$ ) can contribute significantly to the signal recorded with the $171 \AA$ filter (Phillips et al. 2005; Gburek et al. 2006). This high-temperature radiation can be even higher than a lowtemperature one. The main reason for this behaviour is the spectrum shape near $171 \AA$. For the $10^{6} \mathrm{~K}$ plasma, the $\mathrm{Fe} \mathrm{X}$ line is a dominant line, which almost disappears if temperature exceeds the value of $6 \times 10^{6} \mathrm{~K}$ when another line becomes dominant, namely Fe XX. Due to this complication it is possible that, using a TRACE $171 \AA$ filter, we record a hot (above $10^{7} \mathrm{~K}$ ) component rather than a cold (about $10^{6} \mathrm{~K}$ ) one. This possibly explains the observed similarity between durations of impulsive SXR and EUV brightenings.

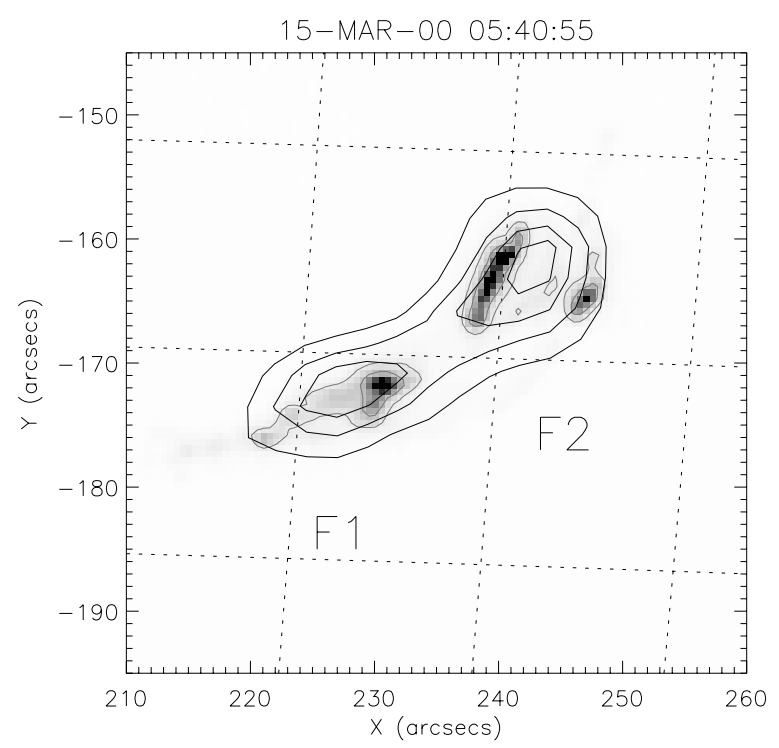

Fig. 3. The image of the 15 Mar. 2000 flare obtained at the maximum of the HXR light curve. The gray intensity scale represents the TRACE $1600 \AA$ emission distribution. Black contours - HXR emission at 30 , 50,70 , and $90 \%$ of the maximum value. Gray contours - UV emission at 10 and $30 \%$ of the maximum value.

Analysing the ratio between the FWHM duration of the impulsive EUV brightening and the HXR burst leads to similar conclusions. Obtained values are presented in the Fig. 6 (middle panel). The histogram has no clear maximum but rather two groups of values. More frequent is the group covering the range $1-5(70 \%)$, since the rest $(30 \%)$ of the points have values higher than 6. Again, these values are similar to the ones obtained for the impulsive SXR brightenings. This result supports prior suggestions that impulsive SXR and EUV brightenings are similar, since the layers emitting these types of radiation are located very close to each other.

A significant difference between impulsive SXR and EUV brightenings is observed for their delay with regard to the HXR burst. Values obtained for the time difference between the maximum EUV brightening and the maximum of the HXR burst are presented in the Fig. 6 (right panel). Almost $80 \%$ of them show a delay shorter than $10 \mathrm{~s}$. In Paper I we reported that delays between SXR and HXR responses have a distribution that can be fitted with the Poisson distribution with a characteristic time $t_{\text {char }}$. Although this Poisson distribution is not clearly seen in Fig. 6 because of the low number of points, we did a similar fit and found that the characteristic time of the separation between the HXR and EUV responses is $6.25 \pm 0.5 \mathrm{~s}$. This result is consistent with theoretical models of reaction observed in EUV range (Somov et al. 1981). In these models the maximal flux of EUVs is observed $5 \mathrm{~s}$ after the HXR peak.

The analysis of impulsive EUV brightenings' observational characteristics generally suggests that there are two main effects forming the observed shape of impulsive EUV brightenings. The first is the non-thermal electron beam that is responsible for the fast occurring maximum of EUV radiation. The second is the thermal front connected with close-lying layer having temperatures about $10^{7} \mathrm{~K}$. This front is responsible for observed durations of EUV brightenings which are similar to the values 

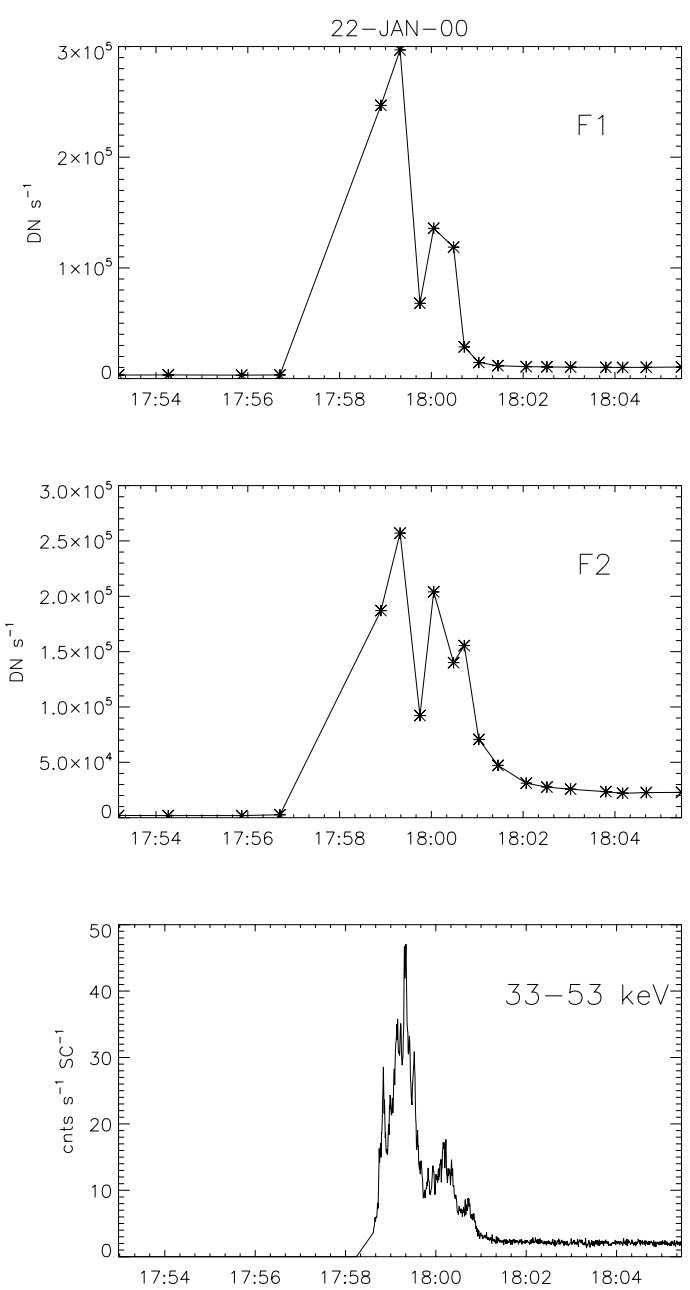

Fig. 4. The TRACE $171 \AA$ light curves for the footpoints seen in Fig. 2 (top and middle panels). The total HXT (M2) light curve (bottom panels).

obtained for hot part of the footpoint emitting in SXR range (Paper I).

\subsection{TRACE 171 Å: quantitative analysis}

Quantitative analysis of the impulsive EUV brightenings was made in a similar way to the impulsive SXR brightenings (Paper I). We excluded HXT L and H channels from this type of analysis. In the $\mathrm{L}$ channel we observed the severe influence of thermal emission, which caused problems with distinguishing footpoints in the image because of loop-top thermal source domination. Strong events are needed for reconstructing the image in the $\mathrm{H}$ channel. We have only two flares (Table 1 ) stronger than moderate $\mathrm{M}$, thus we do not analyse the relation between EUVs and HXRs recorded with the $\mathrm{H}$ channel.

Figure 7 shows the relation between the HXR response (recorded with the M1 channel) and the EUV signal measured in observed footpoints. The calculated correlation coefficient is equal to 0.52 . The value obtained for complete events (for total signals - no spatial distribution) is equal to 0.46 . The correlation is good enough to conclude that both types of emission are produced in the same process - they are caused by non-thermal electron beams.
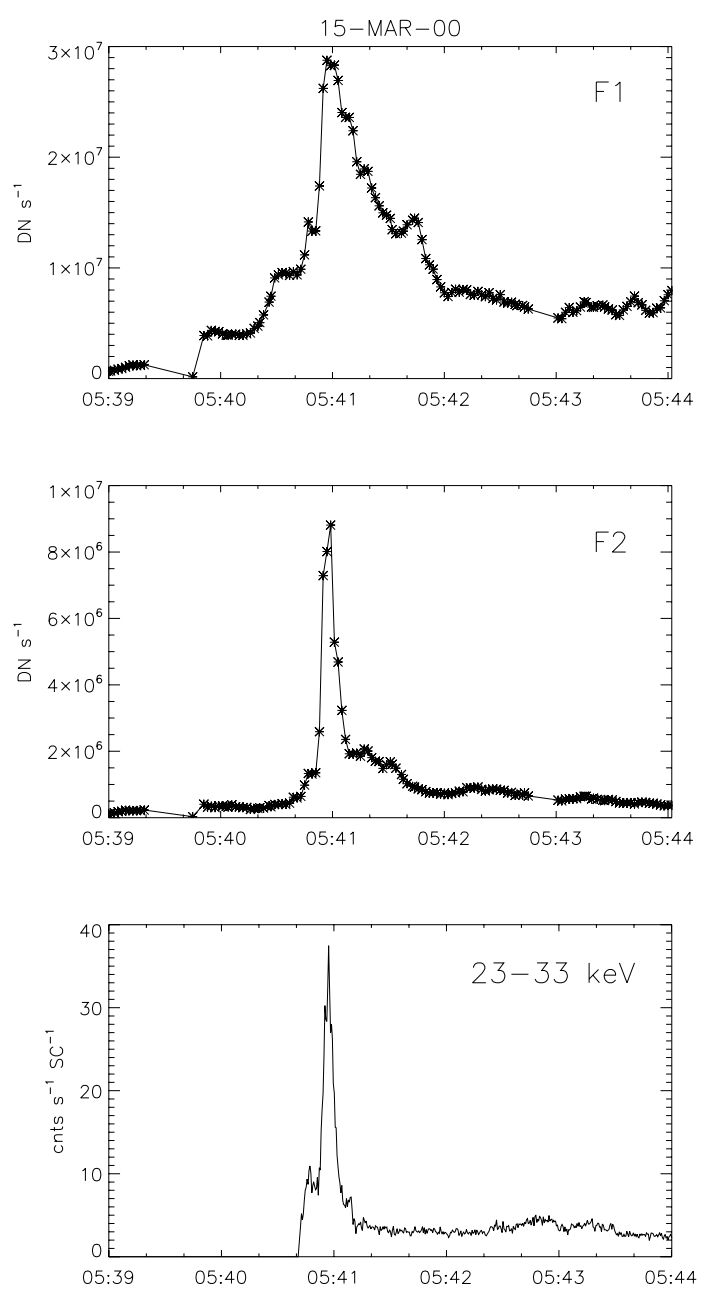

Fig. 5. The TRACE $1600 \AA$ light curves for the footpoints seen in Fig. 3 (top and middle panels). The total HXT (M1) light curve (bottom panel).

Comparison of the relative productivity, $F_{R}$, of EUVs with regard to hard X-rays and power-law indexes taken from single power-law fit is presented in Fig. 8, where $F_{R}$ is defined as a ratio between EUV and HXR fluxes. We observed a lack of the correlation for individual footpoints $(R=0.17)$, as well as for complete events $(R=0.15)$. The correlation coefficients are significantly lower than the ones obtained for the impulsive SXR brightenings (Paper I). This can be considered as an effect connected with the higher energy of the electrons that penetrate the level emitting in EUV range.

This is the next piece of evidence suggesting a similarity between impulsive brightenings observed in the SXR and EUV ranges. The main reason for this could be the density that is relatively low, and even low-energy electrons could penetrate the layer emitting in EUV range. The next two explanations, i.e. thermal front and characteristics of $171 \AA$ filter response have been discussed earlier.

Independently, we tried to estimate the energy of HXR photons that correlates best with observed EUV flux in the following way. Using HXT fluxes obtained in different channels, we were able to calculate parameters of the non-thermal component of the spectrum by using the power-law fit. Hence, with the analytic form of the non-thermal spectrum, we were able to calculate, for a given source, HXR photon flux in several energy 

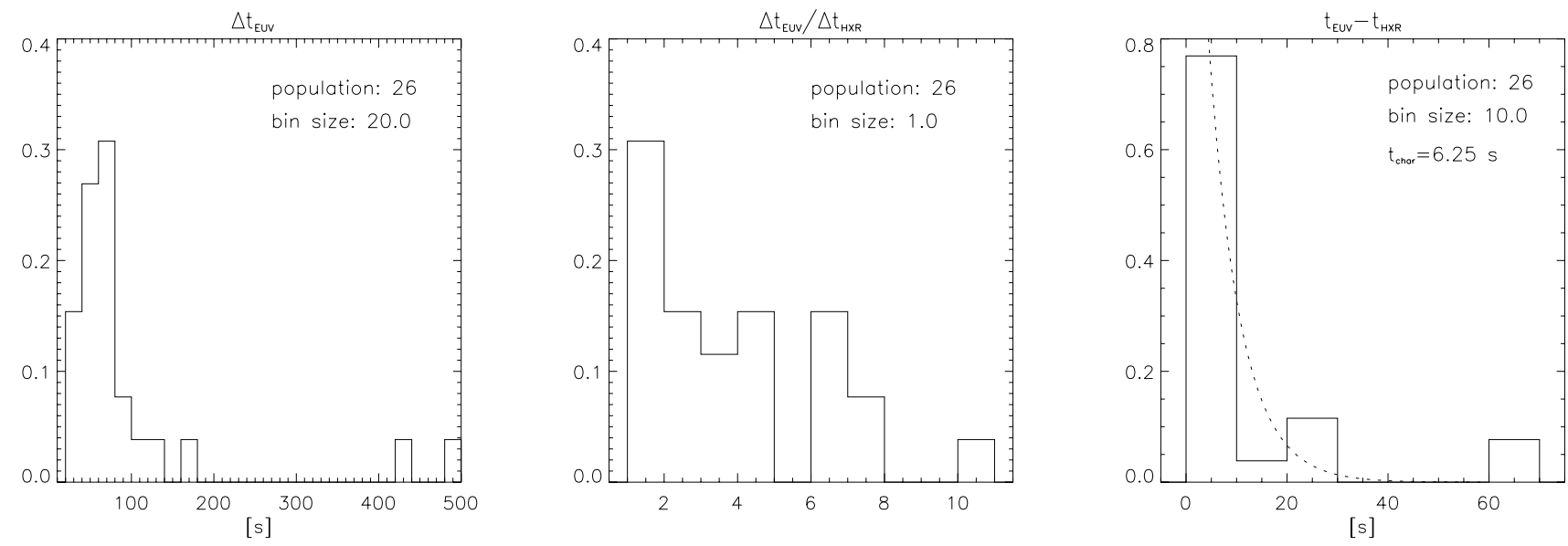

Fig. 6. Normalized histograms of the $F W H M$ duration $\Delta t_{\mathrm{EUV}}$, the ratio $\Delta t_{\mathrm{EUV}} / \Delta t_{\mathrm{HXR}}$, and the EUV/HXR delay for investigated impulsive EUV brightenings. The Poisson distribution fitted to EUV/HXR delay is marked with a dotted line. The characteristic time of this distribution is given in the legend.

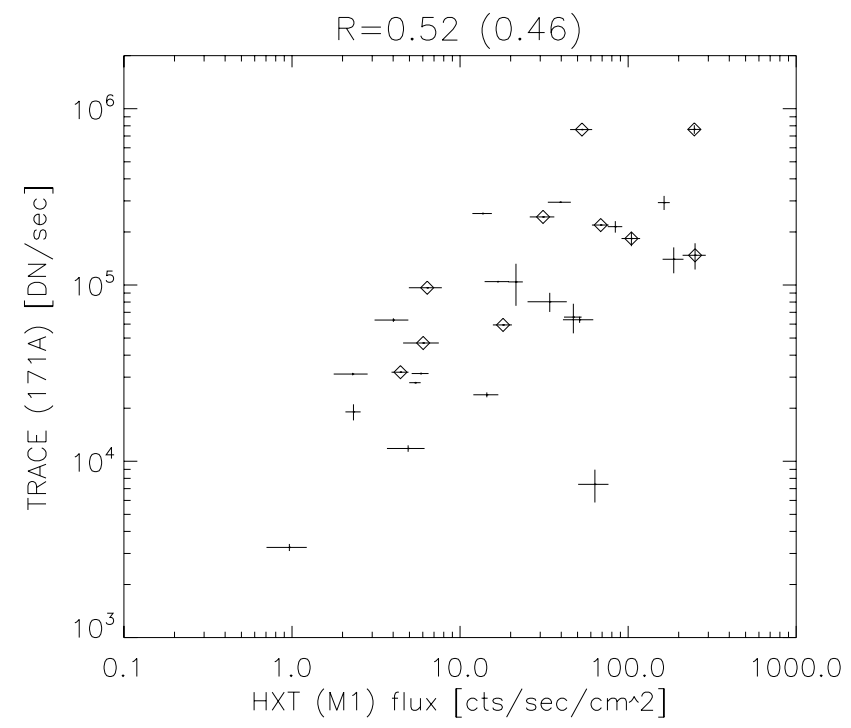

Fig. 7. Relation between fluxes recorded with the HXT (M1) channel and TRACE $171 \AA \AA$. $R$ is the correlation coefficient. Values for the complete events have been marked by diamonds. $R$ for complete events is given in parenthesis.

bands. Next, we made plots similar to Fig. 7, but we used HXR fluxes estimated for consecutive energy values. For each plot, we calculated the energy-dependent correlation coefficient, $R_{\mathrm{E}}$. Figure 9 presents the change of the $R_{\mathrm{E}}$ coefficient with energy. It is seen that the correlation rises fast in the $5-15 \mathrm{keV}$ range. Next, a relatively wide peak is observed with a maximum value equal to $22 \mathrm{keV}$. For higher energies, this correlation weakens again. Despite this wide peak, we conclude that the maximum correlation is observed for higher energy than was observed for SXRs (Paper I). This is connected with different densities of layers emitting SXRs and EUVs.

Generally speaking, results obtained with the TRACE $171 \AA$ filter are very ambiguous. Excellent spatial resolution is not supported by high temporal resolution. Models of an impulsive EUV reaction suggest that temporal resolution of the order of $1 \mathrm{~s}$ will be enough to separate the actual impulsive reaction caused by the non-thermal electron beam from slowly varying

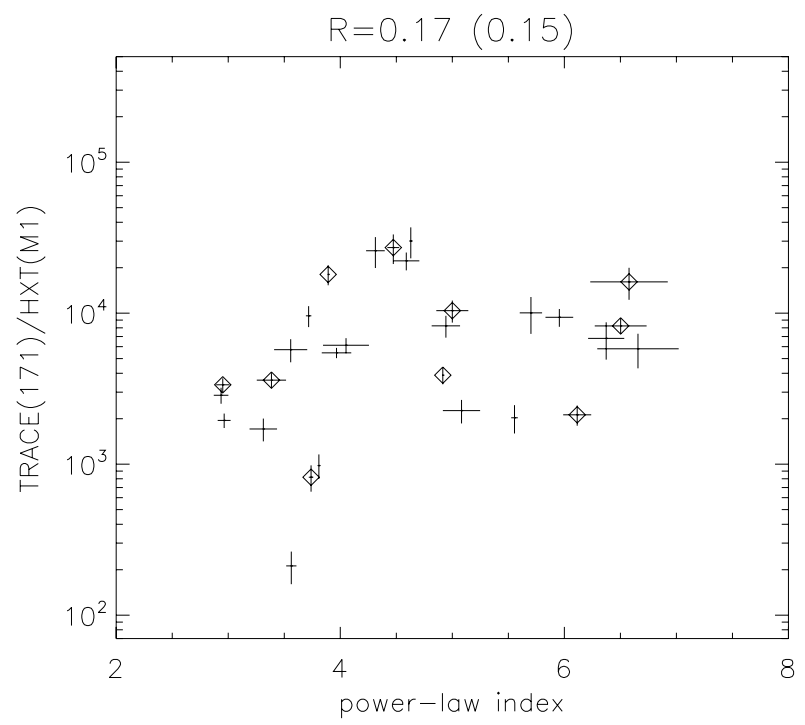

Fig. 8. Plot of the relative productivity, $F_{R}$, of EUV with regard to hard X-rays HXT (M1) and power-law indexes for a single power-law fit. $R$ is the correlation coefficient. Values for the complete events have been marked by diamonds. $R$ for complete events is given in parenthesis.

components connected with conductive heating or back warming. It is possible that huge progress in this field will be made thanks to Hinode satellite (Shimizu 2002), which started observations in autumn 2006.

\subsection{TRACE 1600 Å: observational characteristics of impulsive UV brightenings}

The investigation of TRACE $171 \AA$ data leads to several ambiguities that are mainly caused by the layer emitting in the EUV range being located near the hot plasma emitting SXR radiation. Thus, we had to include observations from the deeper layer of the chromosphere where the influence of hot plasma is expected to be minimal. The TRACE $1600 \AA$ data were chosen for the following reasons.

- We found that observations made with this filter often have a cadence of $2 \mathrm{~s}$. Such high temporal resolution of 


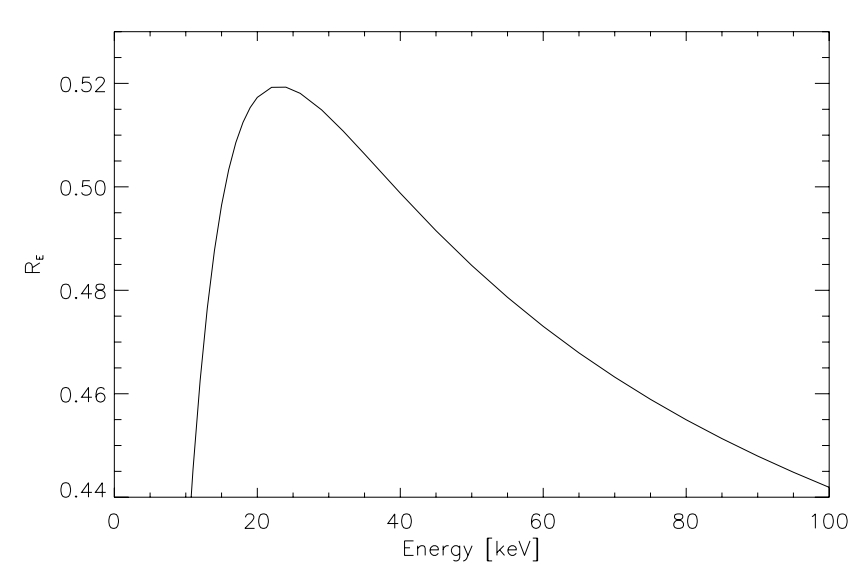

Fig. 9. Energy-dependent correlation coefficient calculated between observed EUV flux and HXR flux calculated for consecutive energy values with the use of a power-law fit to HXT data.

observations is very useful since we compare them to HXR lightcurves that have a $0.5 \mathrm{~s}$ temporal resolution.

- Careful analysis of the TRACE $1600 \AA$ filter response function (Uitenbroek 2004; Fossum \& Carlsson 2005) shows that with this filter we record emission produced at the height of $450 \pm 100 \mathrm{~km}$ above the base of the photosphere.

- This height is related to the temperature minimum region. This is crucial, since we expect that heating connected with the conduction or waves should be weakened in the maximum radiation loss region (Rosner et al. 1978).

We could not desaturate TRACE $1600 \AA$ images, so we were able to obtain observational characteristics of impulsive UV brightenings for only six flares. However, high temporal resolution of these data enabled us to find several independent brightenings in each footpoint. Thus, we obtained observational characteristics for 42 impulsive UV brightenings, which enabled statistical analysis of them.

Figure 10 (left panel) presents the FWHM duration of the impulsive UV brightenings obtained for unsaturated events listed in Table 1 . Over $70 \%$ of brightenings showed durations in the interval of 4-12 s. It means that impulsive brightenings observed with the TRACE $1600 \AA$ filter are significantly shorter than the ones in EUV or SXR. Their clear impulsive character suggests that the reaction in UV is produced directly by the non-thermal electron beams.

This statement is confirmed by analysis of two next observational characteristics. The histogram presenting a relative duration of the impulsive UV brightening and the HXR burst (Fig. 10, middle panel) has clear maximum for the values from 1 to 2 (almost $70 \%$ ). This means that the impulsive UV brightening duration is comparable to the duration of the HXR burst.

Energy deposited in the lower chromosphere by non-thermal electrons induce fast brightenings observed with the $1600 \AA$ filter. In Fig. 10 (right panel), we present a time difference between the maxima of the UV and the HXR pulses. Almost $60 \%$ of the brightenigs reached their maximum less than $2 \mathrm{~s}$ after the maximum of the corresponding HXR peak. Several events showed longer delays, but the values of the delays were always below $10 \mathrm{~s}$. The Poisson fit to the histogram gives the characteristic time of reaction equal to $1.5 \pm 0.3 \mathrm{~s}$.

The result, as mentioned above, was verified independently by analysing observations made for the 2000 Aug. 18 flare. For this event we obtained HXR lightcurves of each footpoint with temporal resolution of $2 \mathrm{~s}$ and compared them to the corresponding UV lightcurves. The comparision of lightcurves obtained for the northern footpoint is presented in Fig. 11. For better presentation, the HXR lightcurve has been shifted vertically by multiplying it by a factor of $5 \times 10^{5}$. The calculated cross-correlation has a maximal value of 0.86 for the shift between curves equal to $0.5 \mathrm{~s}$. A similar result was obtained for the southern footpoint, a cross-correlation have maximal value of 0.81 for the shift equal to $0.4 \mathrm{~s}$.

\subsection{TRACE 1600 Å: quantitative analysis}

Observational characteristics of the impulsive UV brightenings observed in solar flare footpoints show that there is a clear similarity between UV and HXR pulses recorded during the impulsive phase. This is strong evidence that both types of reaction are produced by non-thermal electron beams. The quantitative analysis was made for non-saturated (in $1600 \AA$ filter) footpoints revealing HXR emission strong enough to reconstruct images in the HXT M2 channel, at least.

In summary we found 16 non-saturated sources showing impulsive UV brightenings that were correlated with HXR emission sources. Figure 12 presents the relation between both types of emission. Plots were made for HXT M1 channel. Correlation between observed fluxes is high and equal to 0.67.

For the HXR and SXR flux comparison (Paper I) it was found that points representing individual footpoints of the same event are often situated along a line that is almost perpendicular to the general trend. This effect could be seen in images where one footpoint that is brighter in SXRs shows the lower HXR emission, while the second footpoint seen fainter in SXRs shows the stronger HXR emission. We did not observe such an anti correlation for the footpoints observed with the HXT and TRACE $1600 \AA$.

Strong events observed in the TRACE $1600 \AA$ are saturated. Using the observed relation between UV and HXR fluxes, we tried to estimate the actual UV signal for saturated footpoints. We made a linear fit to the observed relation between HXR and UV fluxes. The fit was made for points representing individual footpoints. Saturated points were shifted vertically to this fit, assuming the HXR flux did not introduce any additional scatter (Fig. 12). Estimated fluxes are even ten times greater than obtained from original saturated images.

Figure 13 presents the relation between $F_{R}$ and the $\gamma$ index of the power-law fit to the HXR flux. The correlation coefficients were calculated for all values except the encircled ones. These points are from the event No. 6 (Table 1), which has very weak HXR emission measured in footpoints. This is the source of large errors, since $F_{R}$ is defined as a ratio of fluxes. Thus, the result obtained for low values of HXR flux should be interpreted very carefully.

The relation between $F_{R}$ of UVs with regard to HXR and $\gamma$ shows a negative correlation between these observables. It is completely different behaviour than we observed for the relative productivity of SXRs with regard to HXRs (Paper I). This difference can be explained in the following way. Let us consider two streams of electrons characterised by power-law distributions with different $\gamma$ indexes, but with the same total energy flux. In the first stream (softer one - high value of $\gamma$ ), more energy is contained in the low-energy electrons that are stopped mainly in the low corona. Hence we observe a strong reaction in SXRs and a relatively weak one in UV range. The second stream 

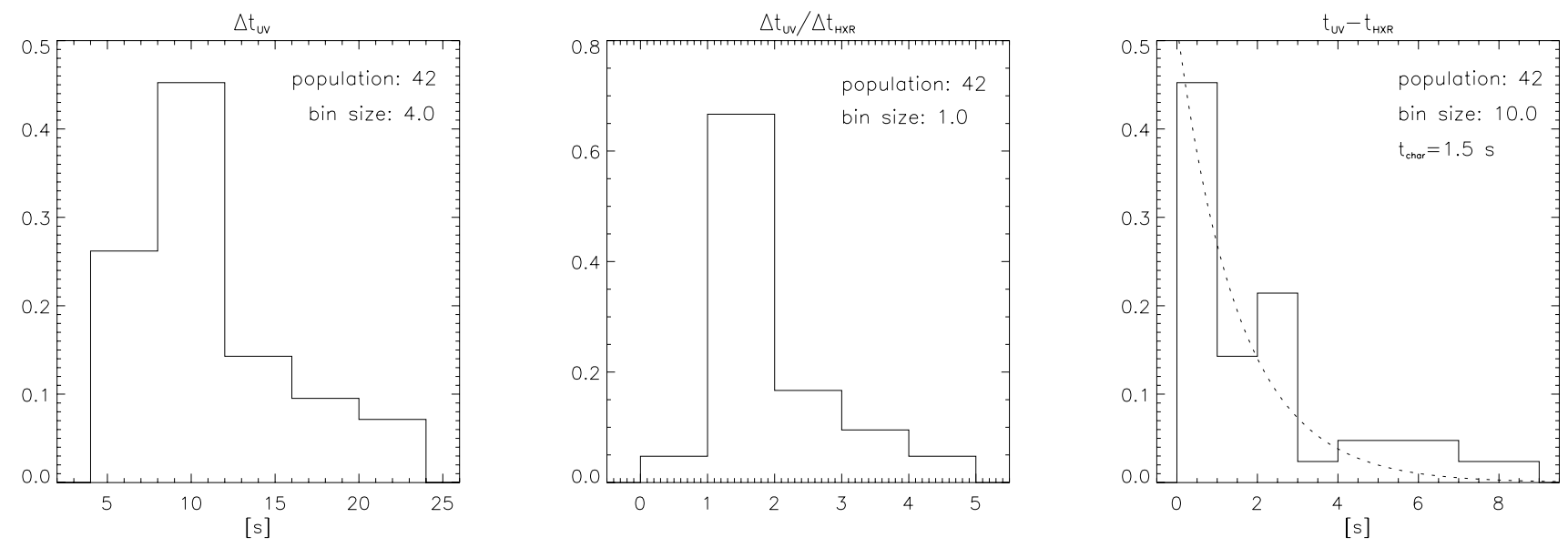

Fig. 10. Normalized histograms of the $F W H M$ duration $\Delta t_{\mathrm{UV}}$, the ratio $\Delta t_{\mathrm{UV}} / \Delta t_{\mathrm{HXR}}$, and the UV/HXR delay for inverstigated impulsive UV brightenings. The Poisson distribution fitted to UV/HXR delay is marked with a dotted line. The characteristic time of this distribution is given in the legend.

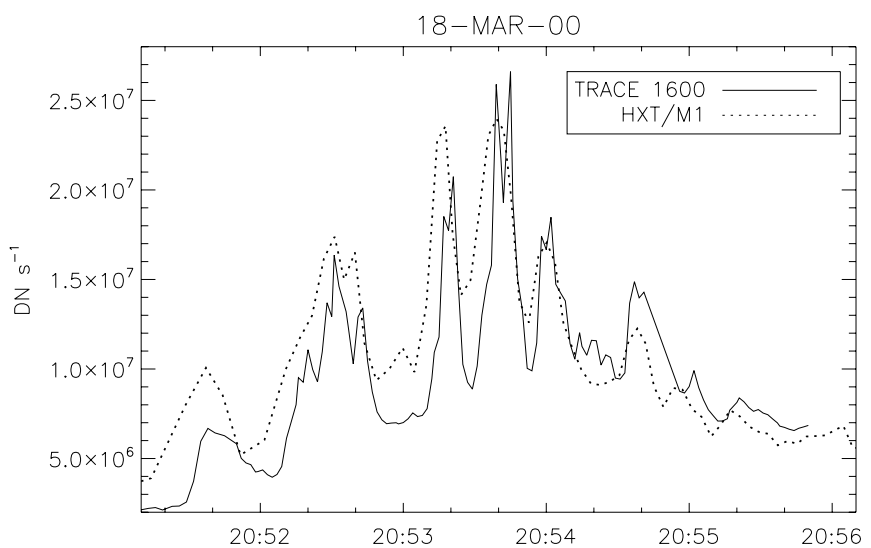

Fig. 11. The UV lightcurve for the northern footpoint of the 2000 Mar. 18 event compared with HXR lightcurve obtained for this footpoint using HXT/M1 images reconstructed with a 2 s cadence. The HXR lightcurve has been multiplied by the $5 \times 10^{5}$ factor for the better presentation.

(harder one - low value of $\gamma$ ) have relatively more energy contained in high energy electrons that could penetrate deep into the chromosphere. In this case we observe a weaker reaction in SXRs and stronger brightening in the UV range. In other words, we observe that the relative productivity $F_{R}$ of SXRs is positively correlated with the $\gamma$ index, whereas the relative productivity $F_{R}$ of UVs is correlated negatively with $\gamma$.

There is a lack of events with low values of gamma, which would confirm negative correlation between the UV relative productivity and gamma. This is because usually such events were the stronger ones and were saturated in the TRACE $1600 \AA \mathrm{im}$ ages. Nevertheless, we tried to estimate an actual UV signal from two saturated events (flares Nos. 4 and 11 from Table 1) as discussed earlier. The estimated UV fluxes were used to calculate $F_{R}$ (Fig. 13) They are located significantly above points representing relative productivity of UVs with regard to HXRs recorded with the M1 channel. Simultaneously they are associated with low values of gamma. This observation strongly supports a scenario in which UV brightenings are produced by high-energy electrons that are able to penetrate the temperature minimum region.

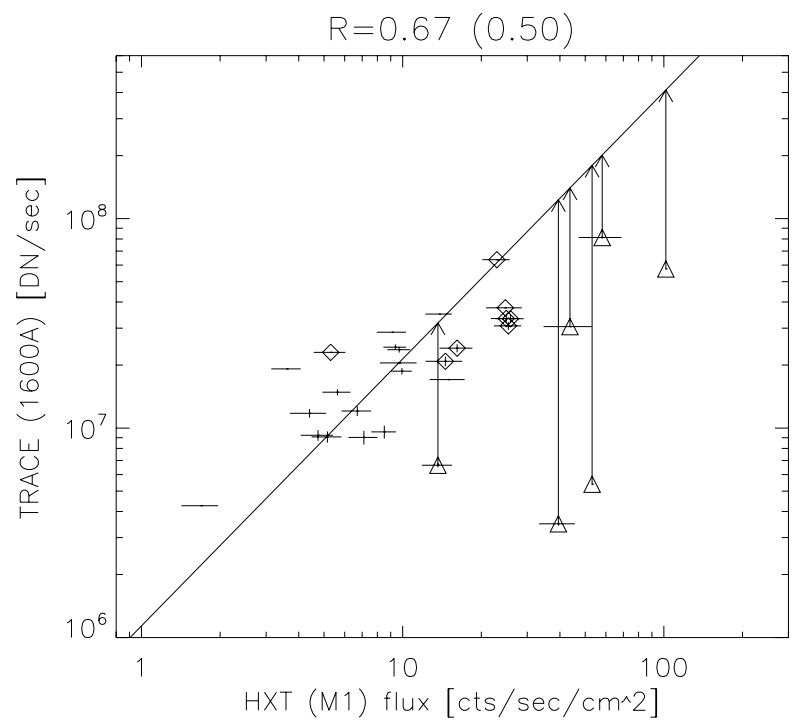

Fig. 12. Relation between fluxes recorded with the HXT/M1 channel and TRACE $1600 \AA$ A. $R$ is the correlation coefficient. Values for the complete events have been marked by diamonds. $R$ for complete events is given in parenthesis. The solid line shows the linear fit to points representing the signal measured in individual footpoints. Saturated points are presented with arrows showing estimation of an actual UV signal.

Again, we independently investigated the change in the correlation between UV and HXR fluxes with energy. The result is presented in Fig. 14. High correlation (about 0.8) is observed for HXR photons with the energy equal to $25 \mathrm{keV}$. This shows that actually impulsive UV brightenings among considered three ranges, i.e. SXR, EUV, and UV, are produced by most energetic electrons.

\section{Summary and conclusions}

Using observations made with TRACE and HXT we obtained several observational characteristics describing the thermal reaction caused by non-thermal electron beams. These characteristics are summarised in Table 3. Moreover, the same characteristics obtained for impulsive SXR (Paper I) brightenings have been included. 


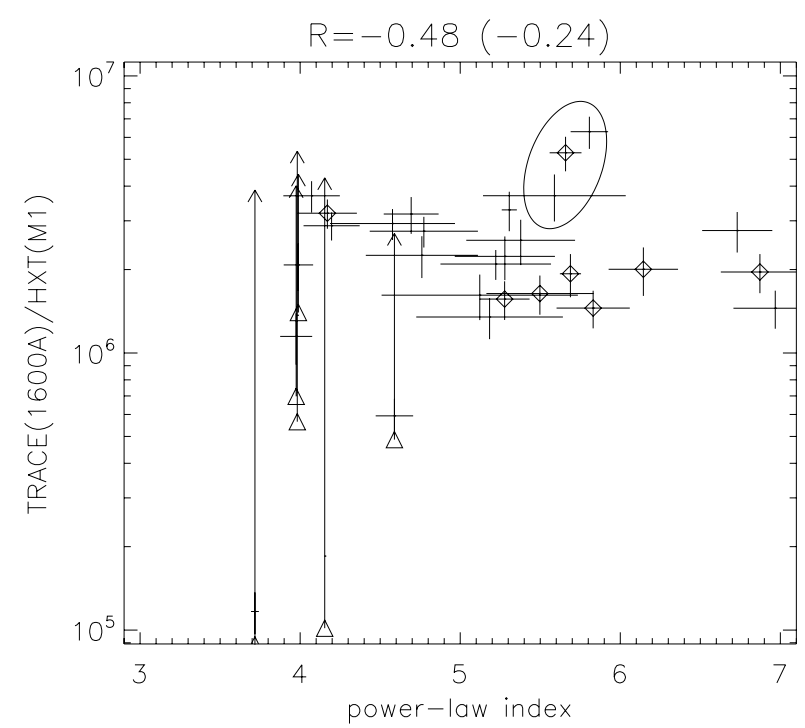

Fig. 13. Plot of the relative productivity of UV with regard to hard X-rays HXT (M1) and power-law indexes taken from single power-law fit. $R$ is the correlation coefficient. Values for the complete events are marked by diamonds. $R$ for complete events is given in parenthesis. Saturated points were shifted using the UV signal estimation presented in Fig. 12.

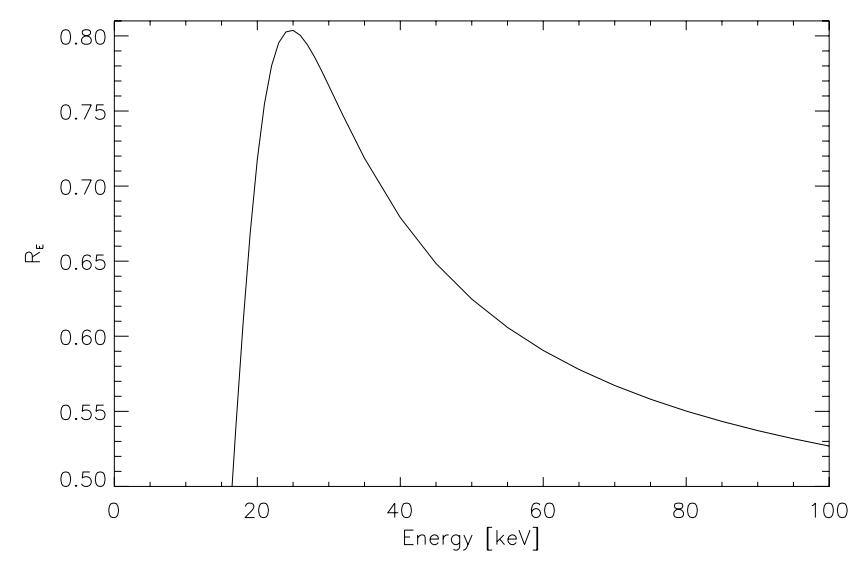

Fig. 14. Energy-dependent correlation coefficient calculated between observed UV flux and HXR flux calculated for consecutive energy values with a power-law fit to HXT data.

The duration of the impulsive brightening is similar in the SXR and EUV. This similarity can be explained in two ways. First, levels of the chromosphere emitting in these wavelength ranges are located very close so one can affect the other. Second, the TRACE $171 \AA$ filter can observe the hot component, about $10^{7} \mathrm{~K}$ (Phillips et al. 2005; Gburek et al. 2006), which is observed usually in the SXR range. The maximum of the impulsive EUV brightening occurs significantly earlier than for the SXR brightening. This result agrees well with the existing models of the EUV reaction in solar flare footpoints.

Significantly different type of behaviour is observed for the impulsive UV brigtenings. They last slightly longer than associated HXR bursts. The time difference between the maxima of the UV brightenings and HXR bursts was very short with a typical value of $1.5 \mathrm{~s}$. For one event, we compared spatially resolved lightcurves of UV and HXR emissions. The result suggests that
Table 3. Impulsive thermal reaction of plasma in solar flare footpoints: observational characteristics of the impulsive brightenings ${ }^{a}$.

\begin{tabular}{|c|c|c|c|}
\hline & $\begin{array}{l}\text { SXT } \\
\text { Al12 }\end{array}$ & $\begin{array}{c}\text { TRACE } \\
171 \AA \\
\end{array}$ & $\begin{array}{l}\text { TRACE } \\
1600 \AA \\
\end{array}$ \\
\hline $\begin{array}{l}F W H M \text { duration } \\
\text { of the impulsive } \\
\text { brightening [s] }\end{array}$ & $\begin{array}{l}20-70 \\
(64 \%)\end{array}$ & $\begin{array}{l}20-80 \\
(73 \%)\end{array}$ & $\begin{array}{c}4-16 \\
(86 \%)\end{array}$ \\
\hline $\begin{array}{l}\text { Relative impulsive } \\
\text { brightening duration } \\
\text { with regard to } \\
\text { the } F W H M \text { duration of } \\
\text { the HXR burst }\end{array}$ & $\begin{array}{c}2-8 \\
(85 \%)\end{array}$ & $\begin{array}{c}1-8 \\
(95 \%)\end{array}$ & $\begin{array}{c}1-3 \\
(84 \%)\end{array}$ \\
\hline $\begin{array}{l}\text { Time difference between } \\
\text { the maximum of } \\
\text { the impulsive brightening } \\
\text { and the maximum of } \\
\text { the HXR burst [s] }\end{array}$ & $\begin{array}{c}0-80 \\
(89 \%)\end{array}$ & $\begin{array}{c}0-30 \\
(92 \%)\end{array}$ & $\begin{array}{c}0-3 \\
(82 \%)\end{array}$ \\
\hline $\begin{array}{l}\text { Characteristic time } \\
\text { of the separation between } \\
\text { thermal and } \\
\text { non-thermal responses } \\
\text { [s] }\end{array}$ & $35 \pm 3$ & $6.25 \pm 0.5$ & $1.5 \pm 0.3$ \\
\hline
\end{tabular}

${ }^{a}$ The percentage of all points that fall into a particular interval are given in brackets.

Table 4. Impulsive thermal reaction of plasma in solar flare footpoints: quantitative analysis.

\begin{tabular}{lccc}
\hline \hline & $\begin{array}{c}\text { SXT } \\
\text { A112 }\end{array}$ & $\begin{array}{c}\text { TRACE } \\
171 \AA\end{array}$ & $\begin{array}{c}\text { TRACE } \\
1600 \AA\end{array}$ \\
\hline $\begin{array}{l}\text { Correlation between } \\
\text { fluxes }\end{array}$ & 0.74 & 0.52 & 0.67 \\
$\begin{array}{l}\text { The range of energy } \\
\text { in which } R_{\mathrm{E}}\end{array}$ & & & \\
$\begin{array}{l}\text { was above 95\% } \\
\text { of the maximal value } \\
{[\text { keV] }}\end{array}$ & $7-40$ & $15-42$ & $22-30$ \\
$\begin{array}{l}\text { Correlation coefficients } \\
\text { between } F_{R} \\
\text { and } \gamma \text { index }\end{array}$ & 0.65 & 0.17 & -0.48 \\
\hline
\end{tabular}

the actual reaction can be even faster, but TRACE $1600 \AA$ images were taken with a cadence of $2 \mathrm{~s}$. This fast reaction strongly supports the opinion that the low chromosphere, close to the temperature minimum region, can be heated by non-thermal electrons.

The quantitative analysis was based on the comparison between reactions observed in EUV, UV, and HXR in flare footpoints. The summary of the results is presented in Table 4. Again, results obtained for impulsive SXR brightenings from Paper I were included. The correlation between fluxes is within the range of $0.52-0.77$, which is enough to conclude that the reaction in SXR, EUV, UV, and HXR is caused by the common physical process - non-thermal electron beams.

The correlation coefficients between the relative productivity $F_{R}$ and the power-law index show significant differences. It can be seen that the lower the level of the atmosphere, the lower the value of the correlation. For the TRACE $1600 \AA$ filter we 
report a correlation close to zero or even an anti correlation, which means that the harder photon flux, the more radiation recorded in the TRACE $1600 \AA$ filter. It can be explained by comparing two electron beams with the same total flux and different slopes. The harder one has relatively more energy contained in high-energy electrons that can penetrate the lower chromosphere, causing enhancement of the relative productivity $F_{R}$ of UV. For the beam characterised with the higher $\gamma$ (softer spectrum), relatively more energy is contained in low-energy electrons that deposit their energy mainly in the SXR emitting region (Tomczak 1999; Mrozek \& Tomczak 2004).

Using parameters $\gamma$ and $A$ from the single power-law fit, we were able to estimate the actual number of HXR photons at a particular energy. We did it for each footpoint and for several energy values. For each monochromatic HXR flux, we calculated correlation coefficient between HXR and EUV or UV flux. There is a clear dependence between the thermal reaction in the flare footpoint at a particular level and the energy of HXR photons; namely, each $R_{\mathrm{E}}$ relation has a maximal value for different energies of HXR photons.

The kind of analysis performed in this paper uses only particular aspects of energy balance in flare footpoints during the impulsive phase. Recently, observations of new satellites, Hinode and $S T e R e O$, together with those derived by SoHO, TRACE, and RHESSI, offer a perfect opportunity to investigate the complete energy balance. It will also be a great challenge for new models of solar flares.

Acknowledgements. The Yohkoh satellite is a project of the Institute of Space and Astronautical Science of Japan. The TRACE satellite is a NASA Small Explorer (SMEX) mission. We would like to thank the anonymous referee for the comments and suggestions, which have improved this paper. This investigation has been supported by a Polish Ministry of Science and High Education, grant No. N203 193733.

\section{References}

Alexander, D., \& Coyner, A. J. 2006, ApJ, 640, 505

Aschwanden, M. J., Brown, J. C., \& Kontar, E. P. 2002, Sol. Phys., 210, 383 Brown, J. C. 1971, Sol. Phys, 18, 489

Cheng, C. C., Vanderveen, K., Orwig, L. E., \& Tandberg-Hanssen, E. 1988, ApJ, 330,480

Deeg, H.-J., \& Ninkov, Z. 1995, Op. Eng. 34, 43

Donnelly, R. F. 1970, ESSA Tech. Rept. No. TR ERL 169-SDL 14

Emslie, G. A., Brown, J. C., \& Donnelly, R. F. 1978, Sol. Phys., 57,175

Farnik, F., Hudson, H., \& Watanabe, T. 1997, A\&A, 320, 620

Fisher, G. H., Canfield, R. C., \& McClymont, A. N. 1985a, ApJ, 289, 414

Fisher, G. H., Canfield, R. C., \& McClymont, A. N. 1985b, ApJ, 289, 425

Fisher, G. H., Canfield, R. C., \& McClymont, A. N. 1985c, ApJ, 289, 434

Fletcher, L., \& Hudson, H. 2001, Sol. Phys., 204, 69

Fossum, A., \& Carlsson, M. 2005, ApJ, 625, 556

Gburek, S., Sylwester, J., \& Martens, P. 2006, Sol. Phys., 239, 531

Handy, B. N., Acton, L. W., \& Kankelborg, C. C. 1999, Sol. Phys., 187, 229

Hudson, H. S., Strong, K. T., Dennis, B. R., et al. 1994, ApJ, 422, L25

Kane, S. R., Frost, K. J., \& Donnelly, R. F. 1979, ApJ, 234, 669

Kosugi, T., Masuda, S., Makishima, K., et al. 1991, Sol. Phys., 136, 17

Kristianpoller, N., \& Dutton, D. 1964, Appl. Opt., 3, 287

Lin, A. C., Nightingale, R. W., \& Tarbell, T. D. 2001, Sol. Phys., 198, 385

Matsushita, K., Masuda, S., Kosugi, T., Inda, M., \& Yaji, K. 1992, PASJ, 44, 89

Mrozek, T. 2006, Adv. Space Res., 38, 962

Mrozek, T., \& Tomczak, M. 2004, A\&A, 415, 377

Nagai, F., \& Emslie, A. G. 1984, ApJ, 279, 896

Ogawara, Y., Takano, T., Kato, T. 1991, Sol. Phys., 136, 1

Phillips, K. J.H., Chifor, C., \& Landi, E. 2005, ApJ, 626, 1110

Rosner, R., Tucker, W. H., \& Vaiana, G. S. 1978, ApJ, 220, 643

Sato, J., Kosugi, T., \& Makishima, K. 1999, Publ. Astron. Soc. Japan, 51, 127

Shimizu, T. 2002, Adv. Space Res., 29, 2009

Somov, B. V., Syrovatskii, S. I., \& Spektor, A. R. 1981, Sol. Phys., 73, 145

Tomczak, M. 1999, A\&A, 342, 583

Uitenbroek, H. 2004, ESA SP-547, 107

Warren, H. P., \& Warshall, A. D. 2001, ApJ, 560, L87 